\title{
"CONTRIBUIÇÃO DA ENFERMAGEM NA ASSISTÊNCIA AOS PACIENTES OBESOS"
}

\author{
Laurinda Madureira Gandolla * \\ Edna Rodrigues ** \\ Olinda Margarida Sanches Varella ***
}

1 - Conceituação: - Primariamente a obesidade pode ser considerada como o desequilíbrio entre ingestão e queima de calorias. Alguns dos estudiosos do assunto ainda a consideram como problema comum das classes abastadas e das sociedades de elevado padrão de vida; isso porque achados históricos revelam que nos períodos de abastança, os casos de obesos eram frequentes em contraposição com os períodos de crises políticas, guerras, cataclismas. Quando os alimentos se tornavam escassos ou de difícil aquisição os casos de obesos também diminuíram. A determinação do excesso de peso é feita por meio de tabelas, se bem que com sérias limitações, visto que fatores biotipológicos devem ser considerados e uma tabela padrão deixaria de ser exata; contudo, os "experts" do assunto partem da premissa de que um padrão de referência peso ideal ou desejável, mesmo na época atual, a tabela ainda é usz tnstrumento valioso, na determinação da obesidade.

Sendo assim. alguns autores definem a obedtade como o excesso de $15 \%$ do peso ideal ou desejável.

Segundo outros, o excesso de peso é consiciando obesidade se atingir $20 \%$ sobre o ideal ou desejável.

Outros há que precisam com exatidão, situando o problema na seguinte escala:

$-20 \%$ sobre peso ideal ou desejável nos adolescentes do sexo feminino;

\footnotetext{
* Supervisora de Enfermagem do Hospital das Clínicas S. P.

* Enfermeira Encarregada do Hospital das Clínicas, S. P.

*** Enfermeira Chefe do Hospital das Clínicas, S. P.
} 
- $25 \%$ sobre peso ideal ou desejável nos adolescentes do sexo masculino;

-- $12 \%$ sobre peso ideal ou desejável na mulher adulta.

- 19 a $29 \%$ no homem adulto.

2 - Etiologia: - Como causa imediata, está o desequilíbrio ingestão e consumo de calorias, visto que científicamente está provado que um grande percentual de obesos não apresenta afecção metabólica de origem endócrina.

Nas pesquisas clínicas realizadas, alguns autores se referem aos obesos neurológicos ou lipofásicos, cujo apetite é estimulado nas áreas hipotalâmicas correspondentes, casos estes mais raramente encontrados, cujo diagnóstico diferencial não é difícil. Disfunção hipofisária, tiroidéa, paratiroidéa nem sempre se associam à obesidade e nem mesmo a síndrome de Stein-Levental (ovário-policístico) que habitualmente é associada à obesidade, existem casos típicos cujo peso é normal.

A obesidade, quando presente nos portadorres de afecção metabólica, pode ser vista como uma consequência e não como causa, pois com insignificante redução no peso, consegue-se melhora considerável no contexto do quadro clínico.

Sabe-se que $50 \%$ dos diabéticos são obesos.

Quando o paciente não é obeso, o aumento do peso reduz a tolerância à glicose, e diminuindo o mesmo a tolerância normaliza.

A inter-ação obesidade-diabetes, sem estar bem definida, pode atuar ccmo elemento intrínseco do problema, visto que propicia o aumento do depósito do panículo adiposo, sem eliminar a afirmativa do desiquilíbrio de ingestão e consumo calórico, sendo possível reduzir o peso do diabético mediante regime dietético adequado. Contudo, vários são os fatores a considerar, dentre os quais enumeramos os seguintes:

\section{3 - Fatores influentes}

3.1 - Metabólicos - tumores que lesam o hipotálamo produzem obesidade; experiências laboratoriais em ratos comprovam que no caso dos tumores em seres humanos, há indução franca à obesidade. Conforme se verifica na experiência de Amaral, cientista norte-amerícano, o centro hipotalâmico da necessidade de ingestão é controlado pelo centro de saciedade que é capaz de inibir o primeiro. A lesão no centro da saciedade leva o animal a uma hiperfagia que o conduz à obesidade.

Os fenômenos da fome e saciedade estão ligados a um componente glicocitário, cujas diferenças de concentração artério-venoso relaciona com a presença ou ausência de concentração gástrica e a sensação da fome. Um índice elevado de glicose coincide com a 
saciedade e na situação inversa, aparecem as manifestações da fome; nos seres humanos observa-se o mesmo fenômeno, após injeção de glucagon.

Há uma interação hipotalâmica, tanto na saciedade como na fome, cuja responsabilidade é atribuída às células glícero-receptoras e o mecanismo glicocitário que paresse ser o fator controle da ingestão diária.

O desequilíbrio ou falha do controle e o mecanismo lipostático cuja ação é inversa, induzem à obesidade (desequilíbrio a longo prazo).

A tese da origem metabólica está para ser afirmada ou afastada, visto que grupos de estudiosos estudam o problema em vários lugares do mundo.

3.2 - Influências familiaress - Em animais de laboratório, já se tem afirmado o fator genético. No homem não se conseguiu afirmar este fator, por causa da impossibilidade de separação dos fatores culturais e genéticos, sendo fácil contudo, notar a correlação entre excesso de peso nos pais e nos seus filhos.

Quando um dos pais é obeso, $40 \%$ dos filhos também são, ao passo que, quando ambos os pais são obesos, a probabilidade dos filhos é de $80 \%$. o que não ocorre com crianças adotadas pelos mesmos pais. Há todavia, a hipótese de uma herança de características dependentes de um fator genético recessivo.

3.3 - Psicológicos: - É fácil constatar o papel relevante dos fatores psicológicos no desequilíbrio da ingestão calórica, mesmo no meio de nossos amigos e parentes para não dizer dos nossos colegas. Achados bibliográficos nos revelam que Freed, cientista norte-americano, em um estudo realizado com 500 obesos, nos seus períodos de preocupação e nervosismo aumentaram até $74 \%$ a ingestão habitual de alimentos. Muitas pessoas têm 0 apetite aumentado, decorrente de frustraçōes e ansiedades, ao inverso daquelas que se a.bstém de alimentos em situaçōes semelhantes. $O$ ato de comer a loda hora pode ser encarado como defesa de frustrações e tensōes nervosas, e é um derivativo agradável.

A obesidade como a magreza é usada sabiamente como arma para se evitarem relações interpessoais indesejáveis e nas situações problemáticas de ordem familiar e doméstica.

A obesidade está ligada à neurose de angústia. Em indivíduos neuróticos a obesidade pode tornar-se definitiva. É fácil constatar como sinônimo de insegurança, o hábito dos prêmios em guloseima, os atos positivos da infância.

3.4 - Atividades físicas: - Nem só a crença popular de que a ausência ou marcante diminuição das atividades físicas levam à obe- 
sidade, há também a base psicológica que mostra a possibilidade de que a inatividade seja a consequência primária, relacionada à teoria metabólica da obesidade.

Nos escolares, o início da obesidade é mais comum no inverno, quando as atividades físicas são reduzidas. As meninas obesas comem menos quantidade que indivíduos normais, porém, gastam $75 \%$ de tempo menos que os meninos em atividades físicas.

A motivação às atividades físicas leva ao decréscimo evidente da gordura somática.

Análises de observações feitas em meninas obesas mostram que elas gastam menos energia nos jogos obrigatórios nas escolas, em comparação com o grupo normal. As atividades desportivas como o sistema de condicionamento físico idealizado por Kenneth $\mathrm{H}$. Kooper, tão em moda no momento, tanto quanto a natação e o pular corda, nos seus mais variados estilos e adaptáveis a todas as idades para indivíduos sadios. quando bem conduzidos, são de considerável relevância para manter as linhas nos níveis normais, no nosso meio na época atual.

3.5 - Influência sócio-econômica: - Um estudo realizado numa grande área urbana demonstra que a obesidade está se acentuando nas classes menos favorecidas, chegando a bater o elevado número de 7 vezes mais, no elemento feminino do que nas classes mais favorecidas. Isto não nos coloca em contraposição ao que dissemos de início, se ponderarmos os fatores seguintes: conceito atual de beleza física; tempo de ocupação; comunicação e publicidade sobre os perigos da obesidade; frequência ao médico sem sintomas objetivos; frequência aos banhos orientados, saunas, psiquiatria e psicoterapia, sem excluir a opção pelos alimentos adequados que são alguns fatores influentes nestes achados ainda em sua fase inicial.

No sexo masculino, a mesma tendência está presente, porém, em proporções consideravelmente mais baixas.

3.6 - Ambiente de hábitos familiares: - Neste particular, gostaríamos de assinalar que a obesidade insĩpiente, pode ser perpetrada, visto que obesos, cujo início se verificou na infância, cujos pais são obesos e com vícios alimentares, uso abusivo de hidrocarbonatos, crença errônea que gordura é sinal de saúde, ingestão desmedida de álcool, são fatores preponderantes no aparecimento da obesidade tanto nas classes modestas como abastadas.

Mudança de regime de vida, como geralmente acontece após o casamento, principalmente em relação ao homem, leva ao aumento progressivo do peso, porque as atividades desportivas são trocadas pela vida sedentária no lar. Em relação à mulher, quando após a gestação, não há o cuidado necessário, aumentam as facilidades ofe- 
recidas pelo ambiente doméstico, o acréscimo de peso ocorrido durante aquele período não regride, a obesidade insipiente pode tornar-se definitiva.

4 - Prevalência: - Se consideramos o que dissemos anteriormente, podemos concluir que os obesos estão presentes em todo o mundo, principalmente nos locais mais desenvolvidos.

Segundo Dr. Medeiros, médico assistente da 1. a Clínica Médica Serviço do Professor Ulhoa Cintra - Hospital das Clínicas da Fasuldade de Medicina da Universidade de São Paulo, em uma lista de 25 moléstias crônicas a obesidade incide 128,9/1000 indivíduos.

5 - Morbidade e Mortalidade - Estatísticas realizadas por comranhias de seguro idôneas. afirmam que tanto a mortalidade com? a morbidade são significativamente mais elevadas nos obesos. É constatado facilmente nos obesos os problemas de colecistopatias crônicas, calculose biliar, varizes dos membros inferiores, osteoartroses, distúrbios mentais, amenoreias e outros distúrbios da menstruação, diabetes Mellitos, areriosclerose, moléstias vasculares e outras.

6 - Incidência: - Estatísticas norte-americanas informam que $50 \%$ dos indivíduos com mais de 40 anos são obesos. No nosso Serviço a obesidade geralmente é evidenciada a partir dos 30 anos. Há porém crianças obesas, geralmente decorrentes de vícios alimentares. Na adolescência não raro se encontram obesos moderados, quando não marcantes. Há quem diga que 12 a $15 \%$ dos adolescentes são obesos de moderados a marcantes. Embora a obesidade seja problema comum das classes média e rica, em nosso meio em grande contingente de trabalhadores em indústrias, principalmente do sexo feminino, são obesos em potencial.

7 - Manifestações Clínicas: - Nos casos moderados, geralmente não há manifestações clínica que possa sugerir uma visita ao médico. Geralmente, quanđo o problemas já se instalou é que o médico é procurado. A consulta clínica está relacionada à situação da saúde na escala de valores para o paciente. Quando o problema interfere mas atividades físicas, limitando-as, o que decorre de um processo lento de adaptação do organismo é que o facultativo é procurado. O que é certo é que a obesidade, ao instalar-se, é acompanhada de fadiga fácil, dispnéia ao esforço, manifestações de deficiência pulmonar, incluindo a síndrome de Pickwick (sonolência acentuada) com frequência aparecem manifestações dermatológicas em caráter periódico ou crônicas, como micoses dos sulcos dos dedos e dobras aa pele. Geralmente estes e os sinais aos quais nos referimos na morbidade são os que levam o paciente à consulta clínica.

Outros procuram o médico por insistência de amigos ou a convite do próprio médico que tem interesse científico no caso. 


\section{8 - ASSISTÊNCIA DA ENFERMAGEM}

\section{1 - Primeiro periodo ambulatorial}

A partir da primeira consulta, o paciente começa a frequentar o hospital para submeter-se aos testes laboratoriais, a fim de serem pesquisados a origem ou a causa desencadente ao problema.

O médico faz os pedidos de exames encaminhando-os, com o paciente, para a enfermeira fazer a orientação e os encaminhamentos necessários. Além dos exames de rotina a todos os pacientes, nos obesos são feitos testes relacionados aos sistemas ou órgãos cuja afecção pode causar obesidade ou aqueles que podem ser lesados por ela.

Naturalmente os dados de bioquímica sanguínea, os referentes ac colesterol, proteínas, taxas de glóbulos, ácido úrico, dosagem de glúcides, lípides totais, função renal e uréia são pesquisados; são incluídos ainda a determinação da glicemia e curva glicêmica.

Geralmente, faz-se avaliação da função tiroideana, mais no sentido de se verificar a possibilidade de administrar-se hormôneo do que de constatar transtornos daquela glândula.

Em casos mais especiais, inclui-se a determinação da função das supra-renais ou genitais.

Após a matrícula do paciente ,a contribuição da enfermagem é constante junto ao paciente, visto que a orientação é feita pela enfermeira.

\section{2-Exames:-}

\section{EXAME}

- Dosagem de colesterol para detectar hipercolisterolemia

- P.B.I. (pesquisa do iodo proteico)
MATERIAL ASSISTENCIA DE ENFERMAGEM

$$
\begin{array}{cl}
\text { Sangue } & \text { - orientação do paciente } \\
8 \text { a 10cc } & \text { - providência do material utili- } \\
& \text { zado } \\
& \text { - colheita de material } \\
& \text { - encaminhamento do material } \\
\text { Paciente } & \text { orientação do paciente } \\
& \text { - administrar a dose de iodo } \\
& \text { - encaminhar o paciente ao labo- } \\
& \text { boratório de P.B.I. } \\
\text { - cuidado especial-restrição total } & \text { de alimentos ou medicamentos } \\
& \text { que contenham iodo, mesmo no } \\
& \text { uso externo. }
\end{array}
$$




\section{EXAME}

- Ceptação do iodo para auxílio no diagnóstico da disfunção tiroideano; procura-se verificar a quantidade de iodo captado pela glândula

- G.T.T. (teste de tolerância à glicose)
- Co G.T.T. (C.T.T. sensibilizado pela cartizona) Para auxiliar diagnóstico de diabetes e pré-diabetes, cortizona diminui a tolerância à glicose.

- I.T.T. (teste de tolerância à insulina)

- Cintilograma (mapeamento da tireóide)

- Colcitologia

- Pelvipneumoperitoneo

- Temperatura basal
MATERIAL ASSISTENCIA DE ENFERMAGEM

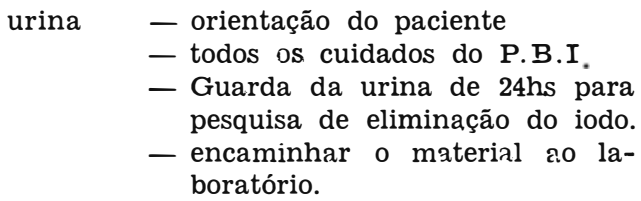

Sangue - orientação do paciente

- supervisionar dieta: 3 dias antes da prova, dieta rica em carbohidratos

- administrar a glicose

- colher 6 amostras de sangue 1 antes de administrar o medicamento e 5 após $30,60,60,120$, e 180 minutos respectivamente.

- peso e mensurações do paciente.

- providência do material utilizado.

- assistir o paciente durante a prova.

- encaminhamento do material ao Laboratório.

Sangue - orientação do paciente

- mesmos do G.T.T.

- administrar as duas doses de cortizona (23:30 e 6hs)

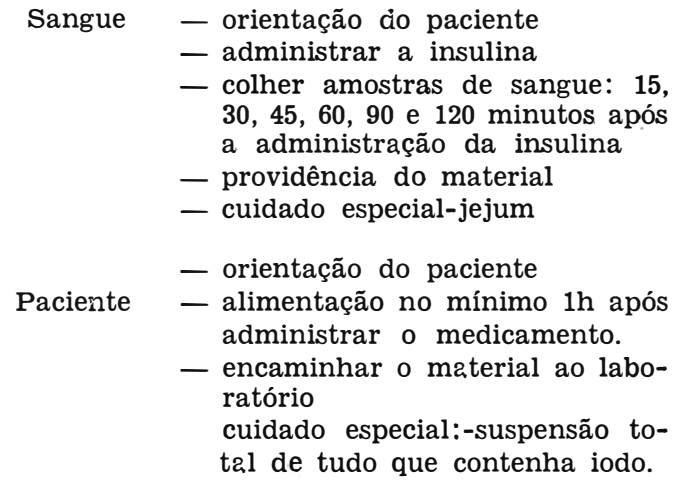
$30,45,60,90$ e 120 minutos após a administração da insulina

- providência do material

- cuidado especial-jejum

- orientação do pacient

Paciente - alimentação no mínimo $1 \mathrm{~h}$ após administrar o medicamento.

- encaminhar o material ao laboratório cuidado especial:-suspensão total de tudo que contenha iodo.

são realizados quando há suspeitas de problema genital 


\section{3 - Período de internação}

Após realizar os testes, o paciente é internado para tratamento, o qual abrange vários aspectos a saber:

Tratamento dietético - Geralmente uma boa proporção dos pacientes respondem bem, e o tratamento dietético dá resultados consideráveis.

As dietas mais usadas no nosso Serviço são as que apresentamos a seguir:

\section{DIETAS EMPREGADAS}

Dieta pobre em hidrocarbohidratos, que baixam para 2200 calorias, empregada durante uma semana, passando para um período de jejum, intermitente e dieta durante um mês. É controlada a glicemia; quando a glicemia chega ao lienar de tolerância pelo jejum, o paciente passa para dieta de 800 calorias até estabelecer o equilíbrio convencionado.

Dieta reduzida $10 \%$ nos carbohidratos associado ao jejum intermitente $0 \cdot$ quanto $o$ paciente suportar controle esporadico de glicemia.

\section{DIETA DE DUNCAN}

Dieta de difícil aceitação é empregada no máximo durante 7 dias porque o paciente não a suporta por mais tempo; consiste de líquidos. Chá sem açúcar, caldo de carne, suco de limão e gelatina com suita ou adocil, obedecendo os níveis calóricos convencionados.

\section{A DIETA É REDUZIDA PARA $10 \%$}

Quando o paciente atingir o peso estabelecido, a dieta passa para $1200,1000,800$ calorias. uma dieta hipocalórica cujo total de calorias foi reduzida para 1009, 30 aproximadamente.

\section{DISTRIBUIÇÃO DAS REFEIÇÕES}

\begin{tabular}{|c|c|c|}
\hline REFEIÇĀO & ALIMENTOS PERMITIDOS & $\begin{array}{c}\text { V.C.T. }=\underset{\text { Calorias }}{\text { Valor total de }} \\
\text { Caloris }\end{array}$ \\
\hline $1 .^{\mathrm{a}}$ & Chá, ovo e laranja & 350 \\
\hline 2. ${ }^{a}$ e $4 .^{a}$ & $\begin{array}{l}\text { batata, bife, abobori- } \\
\text { nha, alface, tomate, } \\
\text { maçã e óleo. }\end{array}$ & 445 \\
\hline $\begin{array}{l}\text { interme- } \\
\text { diárias }\end{array}$ & chá e laranja & 300 \\
\hline
\end{tabular}


Como o paciente respondeu satisfatoriamente e apresentou condições, a dieta passou para 365,53 no V.C.T..*

$\begin{array}{ccc}\text { REFEIÇÃO } & \text { ALIMENTOS PERMITIDOS } & \text { V.C.T. } \\ 1 .{ }^{\mathrm{a}} & \text { maçã } & 100 \\ 2 .^{\mathrm{a}} \text { e } 3 .^{\mathrm{a}} & \text { carne, tomate e alface } & 200,53 \\ \text { interme- } & \text { banana } & 40 \\ \text { diária } & & \end{array}$

Como o paciente respondeu satisfatoriamente e apresentou condições a dieta passou para 365,53 no V. C. T.

Tratamento por atividades físicas

Este tratamento está associado ao tratamento dietético, desde que o paciente apresente condições.

E indicada a prática de exercícios, cabendo aqui a recomendação do método de Cooper, nos casos em que haja indicações deste método.

No nosso Serviço, os pacientes internados recebem orientação complementar do fisioterapeuta e frequentam diariamente a fisioterapia.

\section{Tratamento medicamentoso}

Eventualmente, são utilizadas drogas moderadas do apetite sob rigoroso controle. Associados a estes, são empregados também diurélicos. No nosso Serviço são selecionados para internação, pacientes diabéticos ou com sinais laboratoriais de diabetes química latente, onde então são utilizados também as drogas hipoglicemiantes por via oral.

Em se tratando de pacientes com fatores genéticos, que o tornam "refratárias" ao tratamento dietético, o mesmo é associado ao do jejum. Tanto o tratamento dietético como o jejum podem levar a um deficit de sais minerais e proteínas, sendo então feito $o$ suplemento medicamentoso.

\section{Tratamento pelo jejum intermitente}

Quando se realiza o tratamento dietético com baixa de calorias com boas respostas pelo paciente, é convencionado o jejum intermitente entre os períodos da dieta (dietas n. ${ }^{\circ} 1,2$ e 3 ). Os jejuns são proporcionais às condições do paciente, mas nem sempre a resposta é positiva. Geralmente o paciente adulto suporta de 5 a 7 dias, sendo controlado com pesquisas simultâneas da taxa de glicemia no sangue. 


\section{Tratamento hipnótico}

Este tratamento é associado ao dietético e ao jejum intermitente. Os preconisadores deste tratamento afirmam que, graças à hipnose, o paciente chega a suportar até 30 calorias diárias. (não é realizado no nosso Serviço)

\section{Tratamento psiquiátrico}

É concomitante ao das dietas e jejuns e consiste na matrícula do paciente naquele Serviço, para ser entrevistado periodicamente pelo psiquiatra. Ele trabalha sempre com a enfermeira na identificação de problema psíquicos e como terapeuta de motivação e apoio.

\section{Tratamento cirúrgico}

No nosso serviço este tratamento consiste na realização de dermolipectomias, pois não estamos aparelhados para cirúrgias mais sofisticadas. Quando após os tratamentos anteriores, ficam problemas de estética em virtude a perda de elasticidade dos tecidos, é feita a dermolípetomia, geralmente do abdomen, nádegas, seios, coxas e braços.

Finalmente, mencionamos o método cirúrgico mais sofisticado, indicado como recurso último.

$\mathrm{Na}$ verdade é o recurso derradeiro, usado mais raramente. com indicação muito criteriosa e que consiste na anastomose jejuno ileal. Retira-se aproximadamente $36 \mathrm{cms}$ do jejuno e 10 a $12 \mathrm{~cm}$ do íleo terminal, reduzindo a área de absorção.

O objetivo dessa anastomose é instalar uma síndrome de má ahsorção controlada. Somente nos serviços altamente especializados, $\zeta$ possível realizar esta cirurgia.

Além do nosso Serviço, existem em São Paulo clínicas especializadas para tratamento de obesos, cujos médicos responsáveis são os mesmos do nosso Serviço, e o tratamento utilizado apresenta algumas variações em relação ao nosso Serviço, os resultados também são semelhantes.

A assistência de Enfermagem é intensa e continua durante o internamento do paciente. $\mathrm{E}$ particularmente caracterizada como terapêutica de motivação e apoio, feita por meio de práticas educativas.

\section{9 - Prexaro para Alta}

A permanência do paciente na enfermaria, é variável, pois depende da resposta de cada um ao tratamento. 
Durante todo o tempo de hospitalização o paciente é orientado para que se conduza após a alta clínica, quando continuará o tratamento no domicílio. Quando a alta é assinalada, a ação da enfermeira é intensa no sentido de que a dieta prescrita seja observada, pois muitas vezes, após a alta, o paciente adquire novamente vários quilos do peso perdido no hospital. Quanto ao preparo da dieta, a orientação cabe à nutrição mista; a enfermeira trabalha também no sentido de motivar o paciente às atividades físicas, aos exercícios indicados, aos trabalhos manuais assim como jogos e leituras adequadas. Quanto ao uso de moderadores do apetite, quando prescritos A enfermeira orienta criteriosamente o seu uso.

\section{$10-2 .^{\circ}$ Periodo Ambulatorial}

Esta é a fase de seguimento ambulatorial do paciente após a alta, quando ele frequenta o serviço para controle. O trabalho da enfermeira, nesta fase, é semelhante ao período pré-internação com a agravante da morosidade dos resultados.

Nem sempre c paciente consegue manter o peso adquirido no hospital.

\section{Conclusão:-}

Diríamos que vários fatores responsáveis pela obesidade podem c devem ser controlados; que o tratamento é difícil, prolongado e até inatingível para muitas bolsas, e que os resultados são considerados relativos; que os reflexos da obesidade atingem o indivíduo na sua contextura bio-psico-social; ainda que as possibilidades de controle sejam limitadas, o problema deve ser combatido.

Aqui vai bem o adágio popular: "melhor é prevenir do que remediar".

Em se tratando da obesidade, algumas consequências não podem ser remediadas.

\section{BIBLIOGRAFIA}

1 - ASSIS, Licio Marques de - Aspectos atuais do problema da obesidade. Revista Clinica Geral, 1 (2): 28-38, Fev. 1968.

2 - MEDEIROS Júnior. Geraldo A. de - Obesidade: moléstia metabólica? Revista de Medicina, (Separata), 1972.

3 - DRAMA do obeso começa após a alta. Atualidades Médicas, 5 (3): 42, 1969.

4 - WEST, Edward Stawnton - Text book of biophysical and chemist. 2nd. New York, 1968 p. 356-7.

5 - COOPER, Kennet H. - Teste de Cooper. São Paulo, LEL, 1972. 